\title{
Kuka Youbot Arm Path Planning Based on Gravity
}

\author{
Boquan Zhang a, * and Sheng Gao \\ School of computers, Guangdong University of Technology, Guangzhou 510003, China. \\ a, ${ }^{*}$ 1244818453@qq.com
}

Keywords: mechanical arm; ROS; Motion control; Trajectory planning; Gravitational potential energy.

\begin{abstract}
Aiming at the various algorithms in the mechanical arm trajectory planning, this paper proposes a trajectory planning algorithm based on an open source software platform (ROS) for the control System of the Robot arm. Mechanical arm on the platform, the gravitational potential energy as the optimization criterion, design a kind of optimization algorithm based on polynomial interpolation five times as a planning algorithm, and through the simulation results verify the effectiveness of the improved control algorithm can improve the performance of mechanical arm. This method is of universal guiding significance for the realization of trajectory planning of engineering mechanical arm.
\end{abstract}

\section{Introduction}

Kuka youBot [1, 2] is a state-of-art robot for mobile ma- nipulation research and education. It is also the official platform for Robo Cup@ work competition. Kuka youBot system is made up of an omni-directional mobile platform and a 5-degree-of-freedom arm with a two-finger gripper. Kuka youBot is fully open source control and this gives us an opportunity to make an optimal trajectory planning to improve its performance. During the Robo Cup@work competition and the research works on this platform, we find that the existing kinematics and trajectory planning approach is not satisfied. For example, the moving trajectory of youBot arm from the initial position to the camera ready for detecting position is long and more time is needed. So, proposes a trajectory planning algorithm based on an open source software platform (ROS) for the control System of the Robot arm [1].

The outline of this paper is organized as follows. At first, the forward and inverse kinematics models of the redundant dual-arm robot are given through D-H parameter method in Section 2. Later on, the specific trajectory planning based on gravity are presented in Section 3 Moreover, Section 4 presents a visual simulation and analysis. At last, the conclusions are given in Section 5

\section{Algorithm Description}

\subsection{Trajectory Planning Algorithm Based on Gravitational Potential Energy.}

When given a target position, joints by solving inverse kinematics solution of the corresponding joint solution may be more than one group, depending on the structure of the mechanical arm and each joint can turn Angle range. In most cases, there are multiple sets of corresponding joint angles. Youbot mechanical arm is composed of five series of joints and two fingers in a folder, according to the analysis, the first joint and the fifth joint rotation doesn't change the joint itself relative to the height of the base, so you don't have to consider the two joints, the center of gravity of the change.

From the work space of youbot, you can see that the Angle range of youbot is based on vertical, clockwise and negative. The second joint ranges from $-65^{\circ}$ to $90^{\circ}$, and the third joint range is $-151^{\circ}$ to $146^{\circ}$, and the fourth joint is $-102.5^{\circ}$ to $102.5^{\circ}[3]$.

The second joint center of gravity changes:

$$
\Delta \mathrm{H}_{2}=L_{2} / 2^{*}\left|\operatorname{COS} \theta_{2}^{\prime}-\operatorname{COS} \theta_{2}\right|
$$

The third joint center of gravity changes: 


$$
\Delta H_{3}=L / 2^{*}\left|\left(\left|\operatorname{COS}_{3}^{\prime}\right|-\left|\operatorname{COS} \theta_{3}\right|\right)\right|
$$

The fourth joint center of gravity changes:

$$
\Delta H_{4}=L / 2 *\left|\left(\left|\operatorname{COS}_{4}^{\prime}\right|-\left|\operatorname{COS} \theta_{4}\right|\right)\right|
$$

$\Theta$ 'is the Angle value of the target position.

The energy analysis of the motion of the manipulator from the initial position to the target position can be seen from the following:

$$
P \mathrm{t}=\mathrm{m}_{2} g \Delta H_{2}+m_{3} g \Delta H_{3}+\mathrm{m}_{4} g \Delta H_{4}+\mathrm{W}_{\mathrm{f}}
$$

Mechanical arm and each joint quality of length $\mathrm{L}, \mathrm{m}$, before and after the joint rotation speed $\mathrm{v}$ and position of each joint Angle difference can be calculated, so different solutions can be accurately calculated friction is the work done. Because of the machine's better work and longer life, it is obviously best to choose friction for the least amount of work!

\subsection{Trajectory Planning Algorithm of Five Polynomial Interpolation.}

Using cubic polynomials, according to $\mathrm{q} 0, \mathrm{q} 1 \ldots$, qn and determine the trajectory is characterized by the position and speed continuously, but the acceleration discontinuity in spite of cubic polynomial trajectory have identified "smooth", but for some applications of dynamics and the influence of inertial load can produce some unexpected. In order to obtain a continuous [5] trajectory of acceleration, the position and velocity need appropriate initial and termination conditions, and appropriate initial and termination acceleration values are also required. There are six boundary conditions, such as the Angle, speed and acceleration of the starting point, and the interpolation is required with a five-degree polynomial. It can guarantee the continuous acceleration, without the mutation, which is beneficial to prolong the service life of the machine. The general expressions of the five polynomials are as follows:

$$
\theta(t)=a+b t+c t^{2}+d t^{3}+e t^{4}+f t^{5}
$$

The first derivative and second derivative acceleration expressions are as follows:

$$
\begin{gathered}
\theta^{\prime}(t)=b+2 c t+3 d t^{2}+4 e t^{3}+5 f t^{4} \\
\theta^{\prime \prime}(t)=2 c+6 d t+12 c t^{2}+20 f t^{3}
\end{gathered}
$$

There are six constraints:

$$
\left\{\begin{array}{l}
\theta\left(t_{0}\right)=\theta_{0} \\
\theta\left(t_{f}\right)=\theta_{\mathrm{f}} \\
\theta^{\prime}\left(t_{0}\right)=v_{0} \\
\theta^{\prime}\left(t_{f}\right)=v_{f} \\
\theta^{\prime \prime}\left(t_{0}\right)=a_{0} \\
\theta^{\prime \prime}\left(t_{f}\right)=a_{f}
\end{array}\right.
$$

The conditions in (4) are brought into (1), (2), (3). For the convenience of writing, For $\mathrm{T}=\mathrm{tf}-\mathrm{t} 0, \mathrm{~h}=$ $\theta$ f- $\theta 0$, Get the value of each parameter:

$$
\left\{\begin{array}{l}
a=\theta_{0} \\
b=v_{0} \\
c=\frac{1}{2 a_{0}} \\
d=\frac{1}{2 T^{3}}\left[20 h-\left(8 v_{1}+12 v_{0}\right) T-\left(3 a_{0}-a_{1}\right) T^{2}\right] \\
e=\frac{1}{2 T^{4}}\left[-30 h+\left(14 v_{1}+16 v_{0}\right) T+\left(3 a_{0}-2 a_{1}\right) T^{2}\right] \\
f=\frac{1}{2 T^{5}}\left[12 h-6 h\left(v_{1}+v_{0}\right) T+\left(a_{0}-a_{1}\right) T^{2}\right]
\end{array}\right.
$$




\section{Algorithm Simulation Results}

To verify the feasibility of the proposed method, experiments are conducted in simulation. Due to the limited equipment, the real position of the end-effector cannot be measured directly and precisely and video. Here is the desired path equation (note that just the desired position is given here, but we carried out both simulation and real experiment with time-varying orientation [2]. Due to the limitation of this paper, the details will be presented in future paper

In the simulation experiment, the kuka youbot manipulator is adopted in this paper, and the simulation experiment is carried out on the move it platform. First, find the appropriate urdf model file of youbot on the official website, and then open Movelt! Setup Assistant, then configure the robot arm virtual joint, create Planning Groups, set the start position, and so on.

When selecting the planning algorithm, the planning algorithm of gravity planning is selected, and the results are as follows:

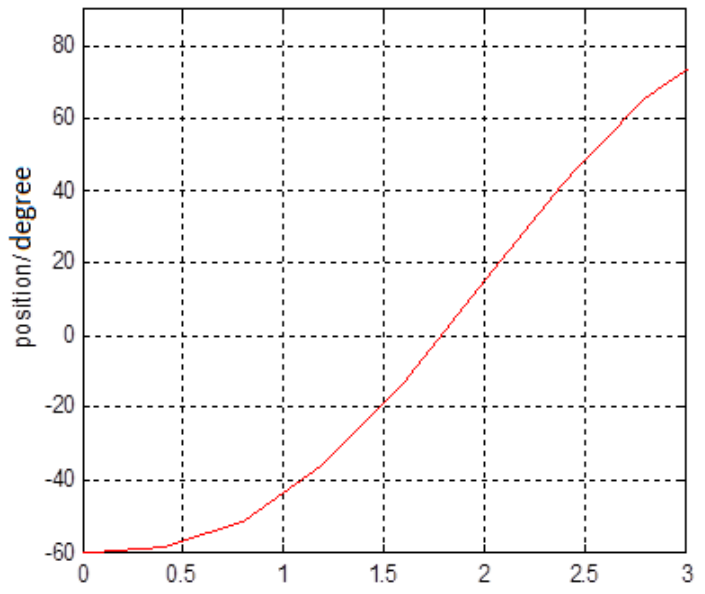

Fig 1. Postion-T

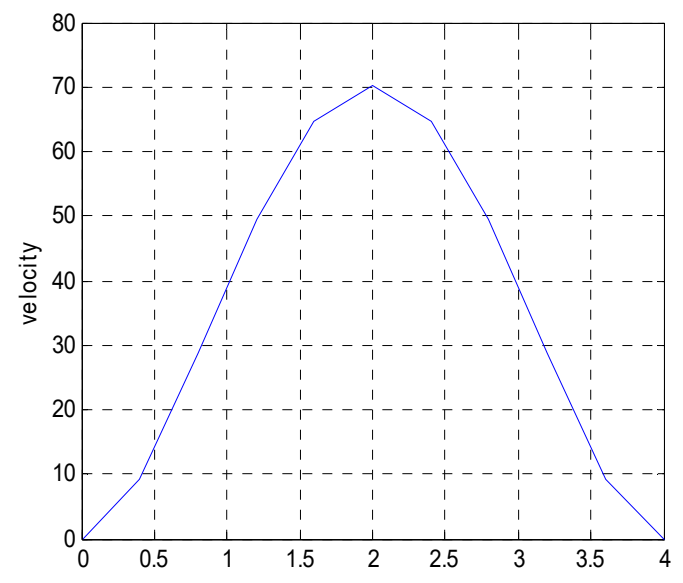

Fig 2. Velocity-T

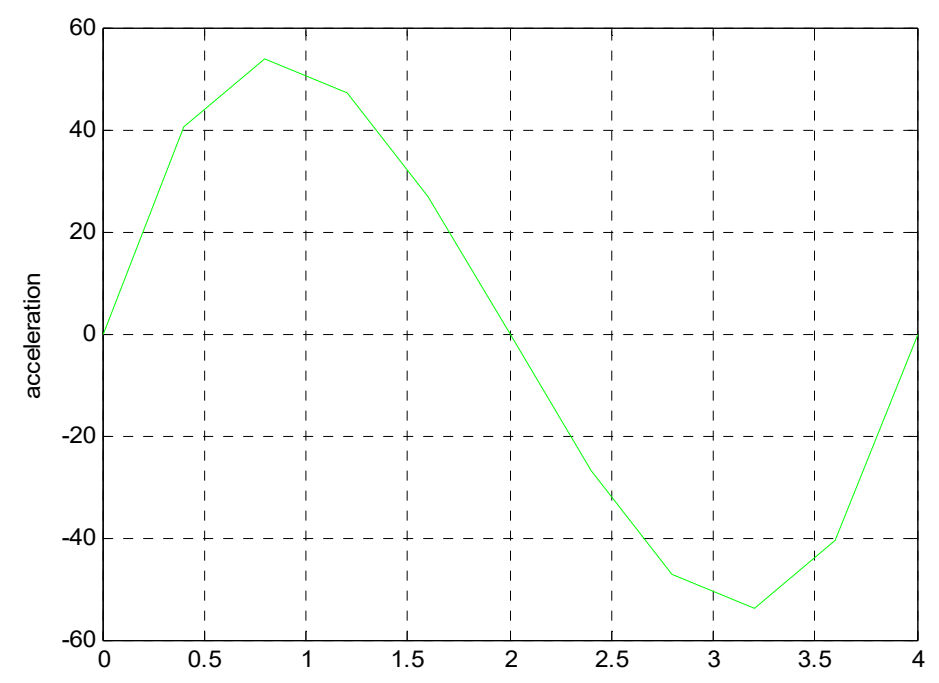

Fig 3. Acceleration-T

As for the figure of simulation and experiment, we just make 102 discrete points in the simulation, while in real experiments, to achieve better performance, 104 is used to obtain the experimental results. According to the experiment, if the more discrete points are adopted, the better performance will be obtained, however, with the same preset value, it will be time-consuming correspondingly. The simulated result is shown in Fig.1, fig 2 and fig 3. While Fig.1 shows the relationship of position and time, it shows that the change in position over time is a smooth continuous curve. And Fig.1 shows the relationship of velocity and time, it shows that the change in velocity over time also is a 
smooth continuous curve. The last, the change in acceleration over time is a smooth continuous curve, it's start with zero and finished also zero.

\section{Conclusion}

In this paper, a strategy based on a gravity and five polynomial interpolation. Method is proposed to solve the inverse kinematics of a 5-DOF manipulator, in addition, the reservation method is also specified, which helps to simplify the complexity of inverse kinematics [4], hence, a complete closed-form solution has been derived. Compared with other methods, the one proposed in this paper does not need to check the calculated solutions, and select and match the optimal solution, and just according to the desired configuration, we can directly obtain the optimal solution without calculating all the possible solutions. Due to the limitation of this paper, we also propose the trajectory planning method to deal with the above-mentioned problems in simulation and experiment, and some remained issues will be given in our future work.

\section{References}

[1]. Zhang L, Zhou C. Kuka youBot arm shortest path planning based on geodesics[C]// IEEE International Conference on Robotics and Biomimetics. IEEE, 2013:2317-2321.

[2]. Fragkopoulos, Christos, Abbas, et al. Comparison of sampling-based motion planning algorithms specialized for robot manipulators [M]. 2012.

[3]. Qiang Yang. Youbot Research on robot trajectory planning. [D]. Guangdong University of Technology, 2015.

[4]. Wei Lin, Wujiang Jiang. Cartesian space trajectory planning for industrial robots. [J]. Mechanical Engineering and Automation, 2014(5):141-143.

[5]. Haoliang Li, Guanfen Liu and Qi Zeng. Youbot. Manipulator kinematics and simulation. [J]. Electromechanical Engineering, 2014(4):18-21. 